\title{
Efficient production of polysaccharide by Chaetomium globosum CGMCC 6882 through co-culture with host plant Gynostemma pentaphyllum
}

\author{
Zichao Wang ${ }^{1}$ - Wenshuo Gao ${ }^{1}$ Xiaoying Liu ${ }^{1} \cdot$ Peizhang Chen ${ }^{1}$. Wenbo Lu $^{1} \cdot$ Fuzhuan Wang ${ }^{1} \cdot$ Haifeng Li ${ }^{1}$ - Qi Sun ${ }^{2}$. \\ Huiru Zhang ${ }^{1}$
}

Received: 27 March 2019 / Accepted: 3 July 2019 / Published online: 18 July 2019

(c) Springer-Verlag GmbH Germany, part of Springer Nature 2019

\begin{abstract}
Endophytic fungus, as a new kind of microbial resources and separated from plants, has attracted increasing attention due to its ability to synthesize the same or similar bioactive secondary metabolites as the host plants. Nevertheless, the effects of the symbiotic relationship between microorganisms and elicitors existed in host plant on metabolite production are not adequately understood. In the present work, the impacts of elicitors (ginseng saponin and puerarin) and symbiotic microorganisms on endophytic fungus Chaetomium globosum CGMCC 6882 synthesizing polysaccharide were evaluated. Results show that the polysaccharide titers increased from 2.36 to $3.88 \mathrm{~g} / \mathrm{L}$ and $3.67 \mathrm{~g} / \mathrm{L}$ with the addition of $16 \mu \mathrm{g} / \mathrm{L}$ ginseng saponin and puerarin, respectively. Moreover, the maximum polysaccharide titer reached $4.55 \mathrm{~g} / \mathrm{L}$ when $C$. globosum CGMCC 6882 was co-cultured with UV-irradiated G. pentaphyllum. This work brings a significant contribution to the research and interpretation of the relationship between endophytic fungus and its host plant.
\end{abstract}

Keywords Endophytic fungus $\cdot$ Host plant $\cdot$ Co-culture $\cdot$ Polysaccharide

\section{Introduction}

With the aggravation of environmental pollution, increase of life stress, increasing occurrences of drug-resistant microorganisms, new cases of life-threatening infections and constant recurrence of diseases, the need for searching novel medicines to treat human diseases has become urgent [1,2]. Generally, there are three approaches for medical scientists to find new pharmacological compounds: first, designing drug purposely towards specific microbial target; second, chemically synthesizing a series of compounds and then screening the promising candidate using microbial tests; third, isolating bioactive compounds from natural biological

Qi Sun

sunqi2017@cqnu.edu.cn

$\triangle$ Huiru Zhang

zhr67@163.com

1 College of Biological Engineering, Henan University of Technology, Zhengzhou 450001, China

2 College of Life Sciences, Chongqing Normal University, Chongqing 401331, China sources $[3,4]$. Although rational drug design through microbial cell platform and synthetic chemistry could significantly shorten the research and development time of a new medicine, insufficient structural complex, restricted structural rigidity, numerous purification and bioactivity tests remained the bottlenecks of these two approaches. Concerning long-term safety, structural complexity and environmental friendliness, extractives from medicinal plants are considered promising new drugs with high biological activities [5].

Endophytic fungi, as a new kind of microbial resources, are normally separated from plants. They have attracted increasing attention due to their ability to synthesize the same or similar secondary metabolites as their hosts, and this offers a new approach for producing bioactive components from medicinal plants [5-7]. It has been reported that $Y e w$ endophytic fungus could produce an anticancer paclitaxel [8], Dendrobium officinale endophytic fungus could produce antitumor and antifungal 4-anilinoquinoline derivatives [9], Passiflora incarnata endophytic fungus could synthesize anti-fatigue and anti-aging flavone chry$\sin$ [10]. Unfortunately, relevant researches so far paid more attention to the bioactivities and biosynthetic pathway of the 
compounds produced from endophytic fungus, whereas the interacting mechanism of the host plants with endophytic fungus is hardly studied [11, 12].

Previously, an endophytic fungus $C$. globosum CGMCC 6882, which was isolated from $G$. pentaphyllum, could produce antibacterial polysaccharide [13]. In this research, $C$. globosum co-cultured with $G$. pentaphyllum and the influence of carbon and nitrogen source, fermentation time, $\mathrm{pH}$ values, particle size of wheat bran and G. pentaphyllum, the addition amount of saponin and puerarin on the synthesis of polysaccharide by $C$. globosum CGMCC 6882 were investigated. Meanwhile, the monosaccharide composition, molecular weight and functional groups of the polysaccharide produced in the co-culture process were analyzed.

\section{Materials and methods}

\section{Microorganism and chemicals}

Chaetomium globosum CGMCC 6882 was isolated from $G$. pentaphyllum herb and stored in China General Microbiological Culture Collection Center (Beijing, China). Chemicals used in this work, unless specified, were analytical grade and purchased from Sinopharm Chemical Reagent Co., Ltd. (Beijing, China).

\section{Seed medium}

Spores of C. globosum CGMCC 6882 were collected by scratching the PDA agar plates (potato $200 \mathrm{~g} / \mathrm{L}$, glucose $20 \mathrm{~g} / \mathrm{L}$ and agar $15 \mathrm{~g} / \mathrm{L}$ ) with a disposable spreader and washing with normal saline. A hemacytometer (Neubauer, Germany) was used to count the number of spores under a phase contrast microscope (Zeiss, Axio Imager 2, Jena, Germany). Then the spore suspension was diluted to a concentration of $1 \times 10^{7}$ spores $/ \mathrm{mL}$ with normal saline and the inoculation volume was $1 \%(\mathrm{~V} / \mathrm{V})$.

\section{Carbon source and nitrogen source}

Starch, maltose, sucrose, glucose, lactose and xylose were used to study the influence of different carbon sources on polysaccharide production. The fermentation medium used for C. globosum CGMCC 6882 culture consisted of $1.5 \mathrm{~g} / \mathrm{L}$ beef extract, $1.5 \mathrm{~g} / \mathrm{L} \mathrm{MgSO}_{4} \cdot 7 \mathrm{H}_{2} \mathrm{O}, 1 \mathrm{~g} / \mathrm{L} \mathrm{KH}_{2} \mathrm{PO}_{4}$, $1 \mathrm{~g} / \mathrm{L} \mathrm{K}_{2} \mathrm{HPO}_{4}$, and $35 \mathrm{~g} / \mathrm{L}$ of a certain kind of the carbon resources selected. Fermentation conditions are set as follows: temperature, $28{ }^{\circ} \mathrm{C}$; cultivation time, $7 \mathrm{~d}$; $\mathrm{pH}$ value of the culture system was maintained at $7.0 \pm 0.02$ with $2 \mathrm{~mol} / \mathrm{L}$ $\mathrm{NaOH}$ and $2 \mathrm{~mol} / \mathrm{L} \mathrm{HCl}$; agitation and aeration were set as $100 \mathrm{rpm}$ and $0.8 \mathrm{vvm}$ and the culture proceeded in a 7.0-L fermenter (BioFlo 115, New Brunswick, USA) with 3.5 L working volume. Different carbon resources and different concentrations of glucose were studied to obtain the optimum glucose concentration for polysaccharide production.

Influence of different nitrogen sources on the polysaccharide production was analyzed using various nitrogen sources, i.e., $\mathrm{NaNO}_{3}(\mathrm{~A}), \mathrm{NH}_{4} \mathrm{NO}_{3}$ (B), $\mathrm{NH}_{4} \mathrm{Cl}(\mathrm{C}),\left(\mathrm{NH}_{4}\right)_{2} \mathrm{SO}_{4}$ (D), $\left(\mathrm{NH}_{4}\right)_{2} \mathrm{CO}_{3}(\mathrm{E})$, ammonia liquor $(\mathrm{F})$, beef extract $(\mathrm{G})$, yeast extract $(\mathrm{H})$, peptone $(\mathrm{I})$ and soybean powder $(\mathrm{J})$. Culture medium and fermentation conditions were the same as above but beef extract was replaced by a certain kind of nitrogen source. Meanwhile, the influence of initial yeast extract concentration on polysaccharide accumulation was further investigated.

\section{Fermentation time and culture medium $\mathrm{pH}$}

Fermentation medium and culture conditions were kept constant, but fermentation time was set as $2,35,7,9,11$ and 13 days to study the influence of fermentation time on the polysaccharide production. After that, the initial $\mathrm{pH}$ of culture medium was regulated to $5,6,7,8,9$ with $2 \mathrm{~mol} / \mathrm{L}$ $\mathrm{NaOH}$ and $2 \mathrm{~mol} / \mathrm{L} \mathrm{HCl}$ to study the influence of medium $\mathrm{pH}$ on the polysaccharide production.

\section{Particle size of wheat bran}

Wheat bran was ground and divided into six meshes (12, $24,50,100,150$ and 200). Then $5 \mathrm{~g} / \mathrm{L}$ of different particle size wheat bran was added into the original culture medium. Inoculation volume and fermentation conditions were the same as above.

\section{Co-culture with G. pentaphyllum herb}

Gynostemma pentaphyllum herb was ground and divided into six meshes (12, 24, 50, 100, 150 and 200). In one group, $5 \mathrm{~g} / \mathrm{L}$ of $G$. pentaphyllum herb with different particle size was added into the original culture medium and sterilized at $121{ }^{\circ} \mathrm{C}$ for $20 \mathrm{~min}$. Meanwhile, another group of G. pentaphyllum herb was irradiated by UV for $24 \mathrm{~h}$ to kill the bacteria on the surface of $G$ pentaphyllum. After the culture medium was sterilized at $121{ }^{\circ} \mathrm{C}$ for $20 \mathrm{~min}$ and cooled to room temperature, irradiated G. pentaphyllum was added into the fermenter through inoculation hole. Inoculation volume, culture medium and fermentation conditions were the same as above.

\section{Addition with saponin and puerarin}

The influence of two bioactive compounds in G. pentaphyllum herb, saponin (i.e., 1, 2, 4, 8, $16 \mu \mathrm{g} / \mathrm{L}$ ) and puerarin (i.e., $1,2,4,8,16 \mu \mathrm{g} / \mathrm{L})$, on the polysaccharide production by $C$. globosum CGMCC 6882 was investigated. 


\section{Polysaccharide titer determination}

At the end of fermentation, culture broth was filtered through a Buchner funnel, the filter liquor was centrifuged at $20,000 \times g$ for $30 \mathrm{~min}$. After that, supernatant $(10 \mathrm{~mL})$ was precipitated with alcohol $(95 \%, 50 \mathrm{~mL})$ at $4{ }^{\circ} \mathrm{C}$ overnight. Achieved precipitates, i.e., the crude polysaccharide extract, were redissolved in $10 \mathrm{~mL}$ deionized water and the content of polysaccharide was determined using phenol-sulfuric acid method at $490 \mathrm{~nm}$.

\section{Polysaccharide extraction and characterization}

The extraction, purification and characterization of polysaccharide produced by C. globosum CGMCC 6882 coculturing with $G$. pentaphyllum were conducted according to the methods described in our previous work [13].

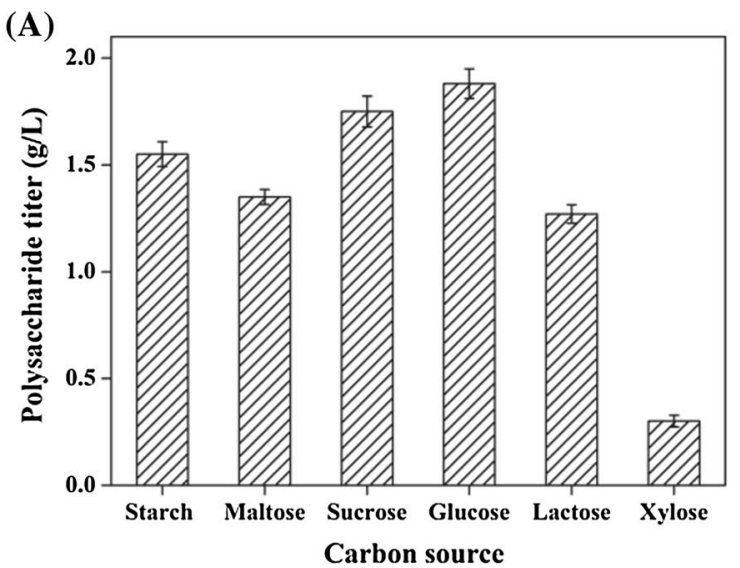

(C)

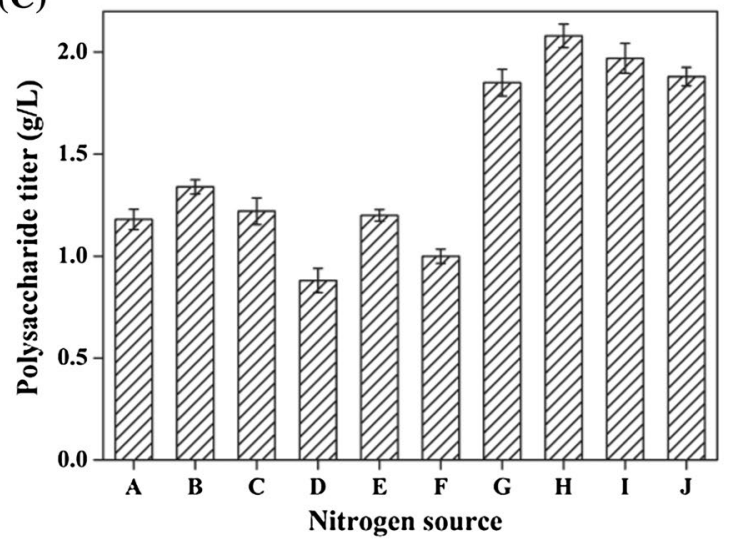

Fig. 1 Effect of carbon source (a), glucose concentration (b), nitrogen source (c) and beef extract concentration (d) on polysaccharide production. The nitrogen sources used in this work are A: $\mathrm{NaNO}_{3}, \mathrm{~B}$ :

\section{Statistical analysis}

All data were expressed as mean \pm standard deviation (SD). One-way ANOVA was performed using the Origin Lab software (Origin Pro 8.5).

\section{Results and discussion}

\section{Carbon source and nitrogen source on polysaccharide production}

Both carbohydrate and nitrogen are major nutrient sources in a culture process, and may significantly affect cell growth and metabolite formation. As shown in Fig. 1a, among all the investigated carbon sources (i.e., starch, maltose, sucrose, glucose, lactose and xylose), glucose is the optimal carbon source for $C$. globosum CGMCC 6882 to synthesize polysaccharide and polysaccharide titer could reach $1.88 \mathrm{~g} / \mathrm{L}$. With the increase of glucose content from 10 to $40 \mathrm{~g} / \mathrm{L}$, polysaccharide titer increased from 1.21 to $2.03 \mathrm{~g} / \mathrm{L}$. However, with

(B)

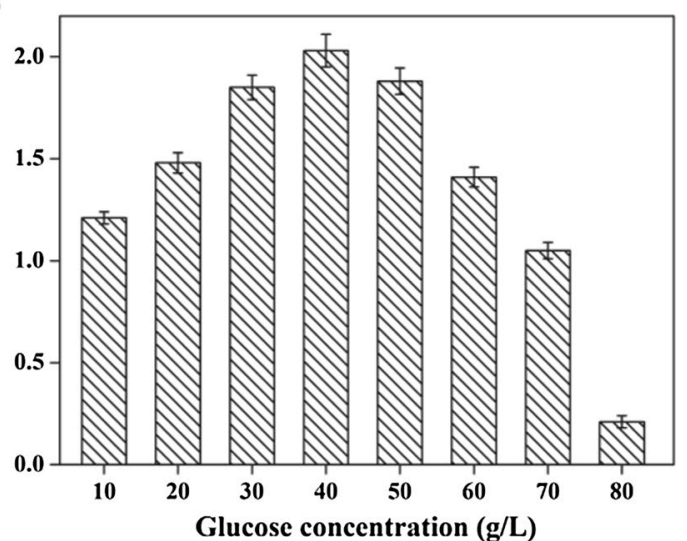

(D)

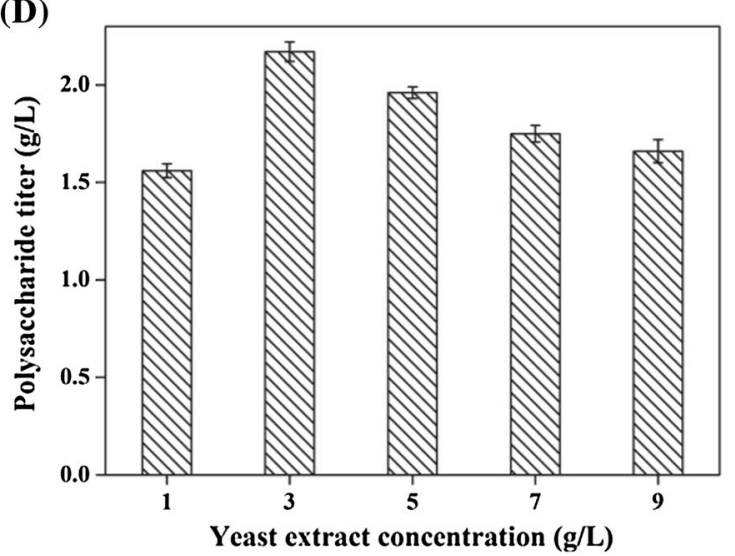

$\mathrm{NH}_{4} \mathrm{NO}_{3}, \mathrm{C}: \mathrm{NH}_{4} \mathrm{Cl}, \mathrm{D}:\left(\mathrm{NH}_{4}\right)_{2} \mathrm{SO}_{4}, \mathrm{E}:\left(\mathrm{NH}_{4}\right)_{2} \mathrm{CO}_{3}, \mathrm{~F}:$ ammonia liquor, G: beef extract, $\mathrm{H}$ : yeast extract, I: peptone and $\mathrm{J}$ : soybean powder 
the concentration of glucose furtherly increased from 50 to $80 \mathrm{~g} / \mathrm{L}$, polysaccharide titer decreased gradually (Fig. 1b). This might be due to the inhibition of cell growth induced by high glucose concentration, and in previous work, we found that the cell growth time was prolonged when glycerol concentration was higher than $40 \mathrm{~g} / \mathrm{L}$ [14]. Meanwhile, in the work reported by Yang et al. [15], glycerol concentration was controlled by fed-batch strategy to reduce the inhibition of high glycerol concentration on cell growth.

As shown in Fig. 1c, the polysaccharide titer produced from C. globosum CGMCC 6882 was significantly influenced by the type and concentration of nitrogen source in the fermentation medium. Among all the studied nitrogen sources (i.e., $\mathrm{NaNO}_{3}, \mathrm{NH}_{4} \mathrm{NO}_{3}, \mathrm{NH}_{4} \mathrm{Cl},\left(\mathrm{NH}_{4}\right)_{2} \mathrm{SO}_{4}$, $\left(\mathrm{NH}_{4}\right)_{2} \mathrm{CO}_{3}$, ammonia liquor, beef extract, yeast extract, peptone and soybean powder), the maximum polysaccharide titer $(2.08 \mathrm{~g} / \mathrm{L})$ was obtained with yeast extract. To obtain a higher polysaccharide yield, the initial yeast extract concentration in the culture medium was further optimized, and the polysaccharide titer reached $2.17 \mathrm{~g} / \mathrm{L}$ when $3 \mathrm{~g} / \mathrm{L}$ yeast extract was added (Fig. 1d). This result is in accordance with previous studies by others. Liu et al. [16] reported that yeast extract was the most favorable nitrogen source for cell growth of Tuber sinense, and the dry cell weight reached $19.53 \pm 0.13 \mathrm{~g} / \mathrm{L}$ with an initial yeast extract concentration at $25 \mathrm{~g} / \mathrm{L}$.

\section{Fermentation time and culture medium $\mathrm{pH}$}

Except for the influence of carbohydrate and nitrogen sources, the culture conditions are also crucial for target compound production $[17,18]$. The influence of fermentation time is shown in Fig. 2a, the polysaccharide titer increased from 0.01 to $2.25 \mathrm{~g} / \mathrm{L}$ from day 2 to day 7 , and decreased gradually to $1.7 \mathrm{~g} / \mathrm{L}$ at the 13 th day. This might be brought by the polysaccharide decomposition for maintaining cell growth when the carbon source was deficient in culture medium [19, 20]. Likewise, Skriptsova et al. [21] also found that the production of water-soluble polysaccharides reached a plateau at around day 7 .

The influence of culture medium $\mathrm{pH}$ is shown in Fig. 2b, from which it could be seen that the maximum polysaccharide titer $(2.36 \mathrm{~g} / \mathrm{L})$ was obtained when the original culture medium $\mathrm{pH}$ was 7.0. Many researchers have also reported the influences of fermentation $\mathrm{pH}$ on target compound production. Jiang et al. [22] reported that alkaline $\mathrm{pH}$ shock could increase Validamycin A production by $27.43 \%$. Wu et al. [23] adopted a two-stage pH-shift control strategy to improve the (2S)-pinocembrin production from D-glucose by engineered Escherichia coli. Some studies revealed some of the mechanisms of the $\mathrm{pH}$ influence. For instance, Zou et al. [24] found that nitrifying aerobic granular sludge (N-AGS) alkaline fermentation facilitated the carbon source recovery, and N-AGS acidic fermentation benefited the phosphorus recovery.

\section{Co-culture with wheat bran and G. pentaphyllum herb}

Given that fungus forms a pellet during the submerged fermentation process and then affects bacterial growth and target compound synthesis $[25,26]$, wheat bran and $G$. pentaphyllum herb were used as upholder to investigate the fungal morphology on polysaccharide production. As shown in Fig. 3a, polysaccharide titer was $3.18 \mathrm{~g} / \mathrm{L}$ when the particle size of wheat bran was 12 mesh, and the polysaccharide titer decreased with the reduction of wheat bran particle size. Although G. pentaphyllum herb as upholder obtained the similar tendency of polysaccharide production to wheat bran (Fig. 3b), the maximum polysaccharide titers of G. pentaphyllum herb with UV irradiation and sterilization were $4.55 \mathrm{~g} / \mathrm{L}$ and $4.25 \mathrm{~g} / \mathrm{L}$, respectively. Because of the
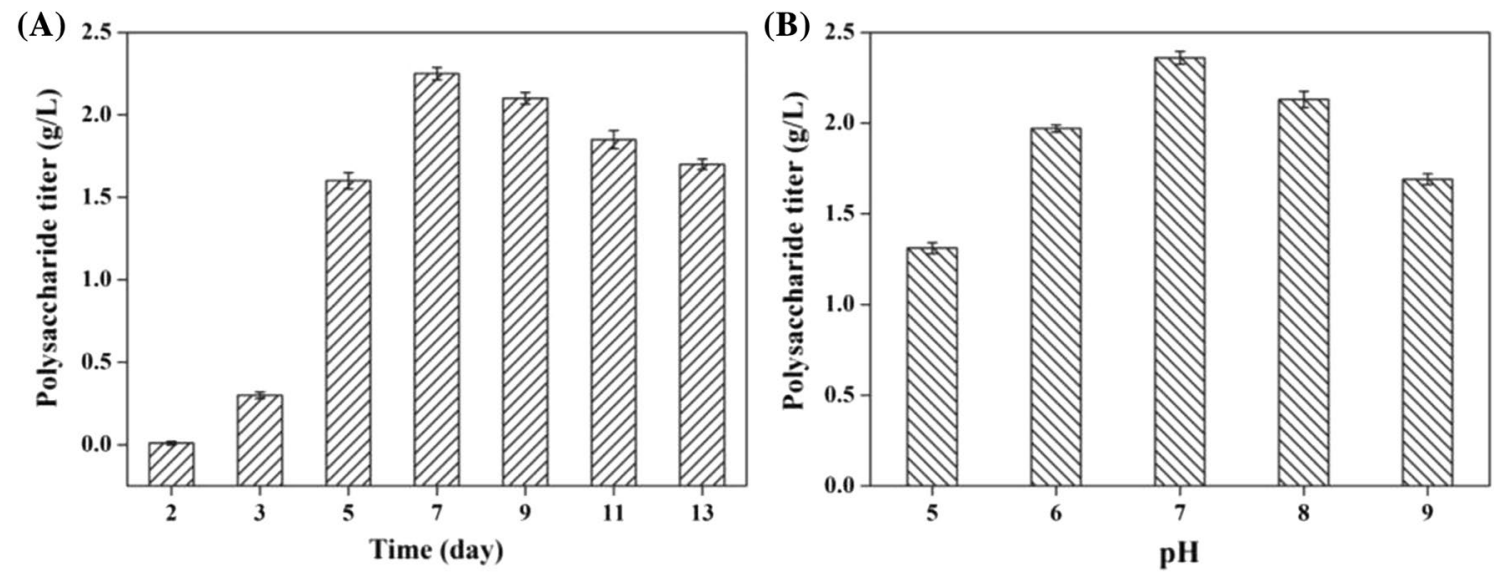

Fig. 2 Fermentation time (a) and culture medium $\mathrm{pH}(\mathbf{b})$ on polysaccharide production 
(A)

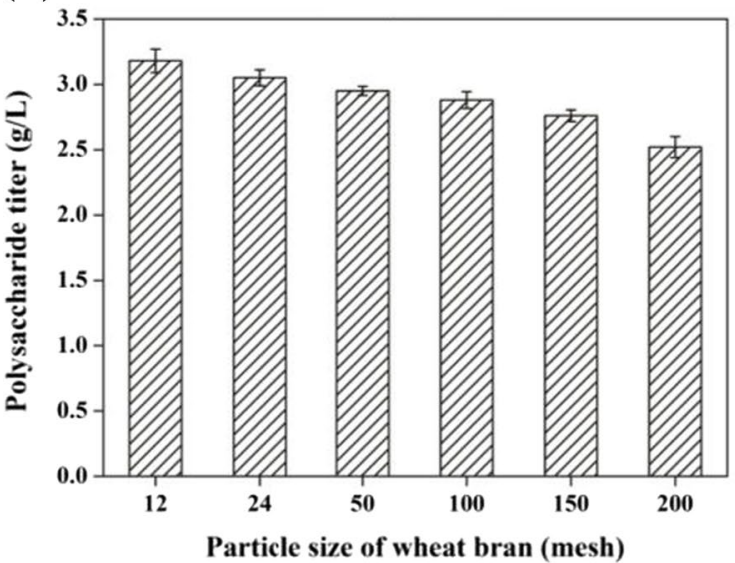

(B)

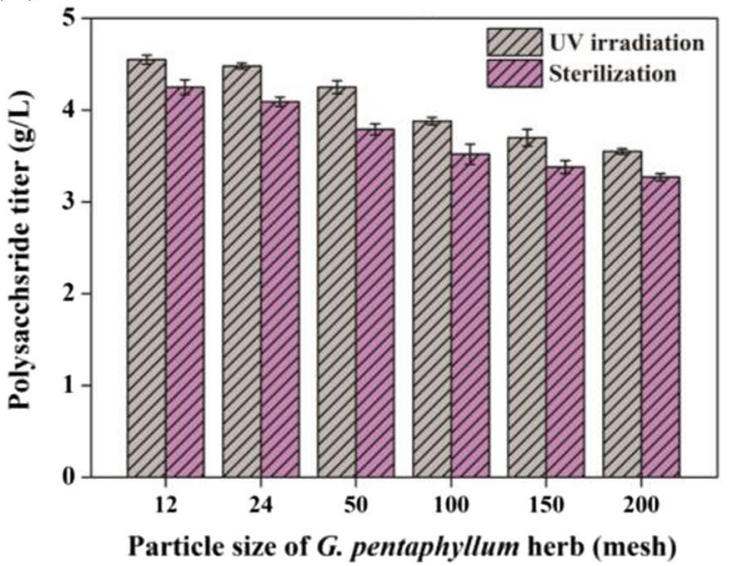

Fig. 3 Particle size of wheat bran (a) and host plant G. pentaphyllum herb (b) on polysaccharide production

low content of bioactive ingredients (including polysaccharide) in plants $[5,6]$, we assume that the polysaccharide in fermentation medium was mainly produced by $C$. globosum CGMCC 6882. At the same time, polysaccharide titers of UV irradiation cases were all higher than those of sterilization. This is possibly due to that G. pentaphyllum herb not only provided upholder for C. globosum CGMCC 6882 growth, but also offered symbiotic environment for polysaccharide synthesis, such as the interacting microorganisms and substances [27, 28]. Therefore, polysaccharide titer of G. pentaphyllum herb group was higher than that of wheat bran, and UV irradiation case was higher than that of the sterilization case.

Two points should be mentioned here regarding the polysaccharide produced by C. globosum CGMCC 6882 in this work. First, glycosides are the major bioactive ingredients and chemical constituents in G. pentaphyllum herb [29], researchers pay more attention to glycosides, but less attention to polysaccharides. Second, C. globosum is mainly studied by researchers for chaetoglobosin A synthesis [30, 31], there are few researches for polysaccharide production by $C$. globosum. Therefore, the current polysaccharide titer by $C$. globosum CGMCC 6882 is hardly compared, but we believe that this titer should not be the highest and will continue to increase in the future through genomic, transcriptomic, and metabonomic investigations.

\section{Elicitors ginseng saponin and puerarin}

Endophytic fungi may produce many bioactive compounds including saponin, flavonoid, polysaccharide, alkaloid and growth regulating agent, to promote host plant growth and help the host plant to keep healthy [32]. Nevertheless, there are few researches on the effects of host plant bioactive compounds as elicitors for endophytic fungus synthesizing bioactive metabolites. Figure $4 \mathrm{a}$ shows that the increase of
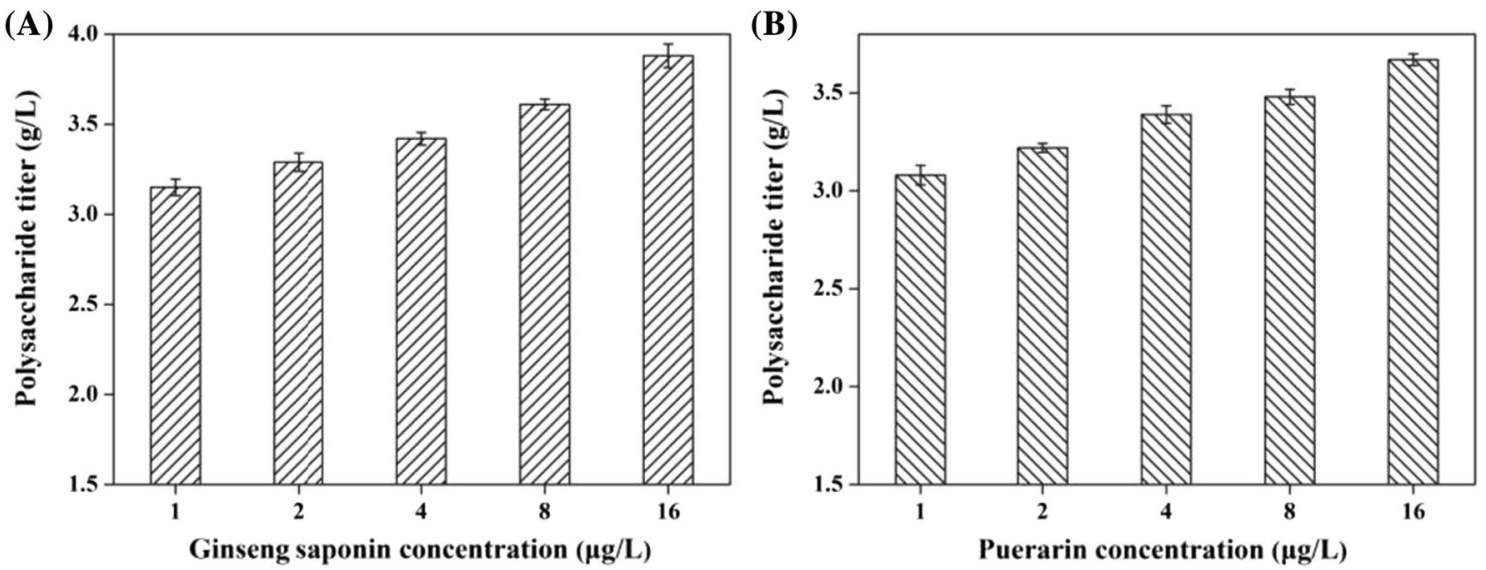

Fig. 4 Elicitors of ginseng saponin (a) and puerarin (b) on polysaccharide production 
polysaccharide titer in fermentation medium was consistent with the addition of ginseng saponin, and the polysaccharide titer could reach $3.88 \mathrm{~g} / \mathrm{L}$ when the ginseng saponin concentration was $16 \mu \mathrm{g} / \mathrm{L}$. Meanwhile, the addition of puerarin acted in the same influencing tendency as ginseng saponin, and the polysaccharide titer was $3.67 \mathrm{~g} / \mathrm{L}$ with the addition of $16 \mu \mathrm{g} / \mathrm{L}$ puerarin (Fig. $4 \mathrm{~b}$ ). This might be that the ginseng saponin and puerarin used in this work enhanced the metabolic process of polysaccharide synthesis in C. globosum CGMCC 6882 [32, 33], and these will be studied in our future work. Meanwhile, many researchers obtained results similar to this work, for instance, Tian et al. [33] found that flavonoids produced by host plant Cephalotaxus harringtonia displayed significant beneficial effects on the hyphal growth of endophytic fungus Paraconiothyrium variabile. Conversely, Shweta et al. [34] demonstrated that camptothecin produced by host plant Nothapodytes nimmoniana (Grahm) Mabb. (Icacinaceae) inhibited the fungal endophyte growth.

\section{Characterization of polysaccharide produced from C. globosum CGMCC 6882 co-culturing with host plant $G$. pentaphyllum herb}

Bioactivities of polysaccharide were affected by its chemical structure including repeating unit, monosaccharide types, glycosidic bond of main chain, chain length, branch degree of chain, flexibility and configuration of chains [35]. In this work, the elution profiles (Fig. 5a) and FT-IR spectroscopy results (Fig. 5b) of polysaccharide produced from C. globosum CGMCC 6882 co-culturing with host plant G. pentaphyllum perfectly matched with the previously published results for antibacterial polysaccharide GCP [13]. Meanwhile, Table 1 shows that the monosaccharide composition, weight-average molecular weight $(\mathrm{Mw})$, number-average
Table 1 Characteristics of polysaccharide produced by C. globosum CGMCC 6882 from co-culture with G. pentaphyllum herb

\begin{tabular}{lll}
\hline Items & GCP & Polysaccharide \\
\hline Arabinose $(\mu \mathrm{mol} / \mathrm{L})$ & 0.64 & 0.58 \\
Galactose $(\mu \mathrm{mol} / \mathrm{L})$ & 2.58 & 2.89 \\
Glucose $(\mu \mathrm{mol} / \mathrm{L})$ & 23.53 & 21.03 \\
Xylose $(\mu \mathrm{mol} / \mathrm{L})$ & 0.90 & 0.67 \\
Mannose $(\mu \mathrm{mol} / \mathrm{L})$ & 2.47 & 1.95 \\
Glucuronic acid $(\mu \mathrm{mol} / \mathrm{L})$ & 0.27 & 1.24 \\
Weight-average molecular weight $(\mathrm{Mw}$, & $8.093 \times 10^{4}$ & $7.904 \times 10^{4}$ \\
$\quad$ Da) & & \\
Number-average molecular weight & $7.982 \times 10^{4}$ & $7.695 \times 10^{4}$ \\
$\quad$ Mn, Da) & & \\
Polydispersity $(\mathrm{Mw} / \mathrm{Mn})$ & 1.014 & 1.027 \\
\hline
\end{tabular}

molecular weight $(\mathrm{Mn})$ and polydispersity $(\mathrm{Mw} / \mathrm{Mn})$ all corresponded with GCP, which further suggested that polysaccharide produced by C. globosum CGMCC 6882 from co-culturing with host plant $G$. pentaphyllum was the same polysaccharide as GCP in our previous work [13].

\section{Conclusions}

This work demonstrated that the production of polysaccharide by an endophytic fungus, C. globosum CGMCC 6882, was not only affected by fermentation conditions, including carbon source, nitrogen source, fermentation time, culture medium $\mathrm{pH}$ and fungal morphology, but also influenced by other symbiotic endophytic fungi in host plant and the compounds produced by host plant, i.e., saponin and puerarin. Our findings bring a significant contribution to the research and interpretation of the relationship between endophytic fungus and its host plant. Moreover, this work provided
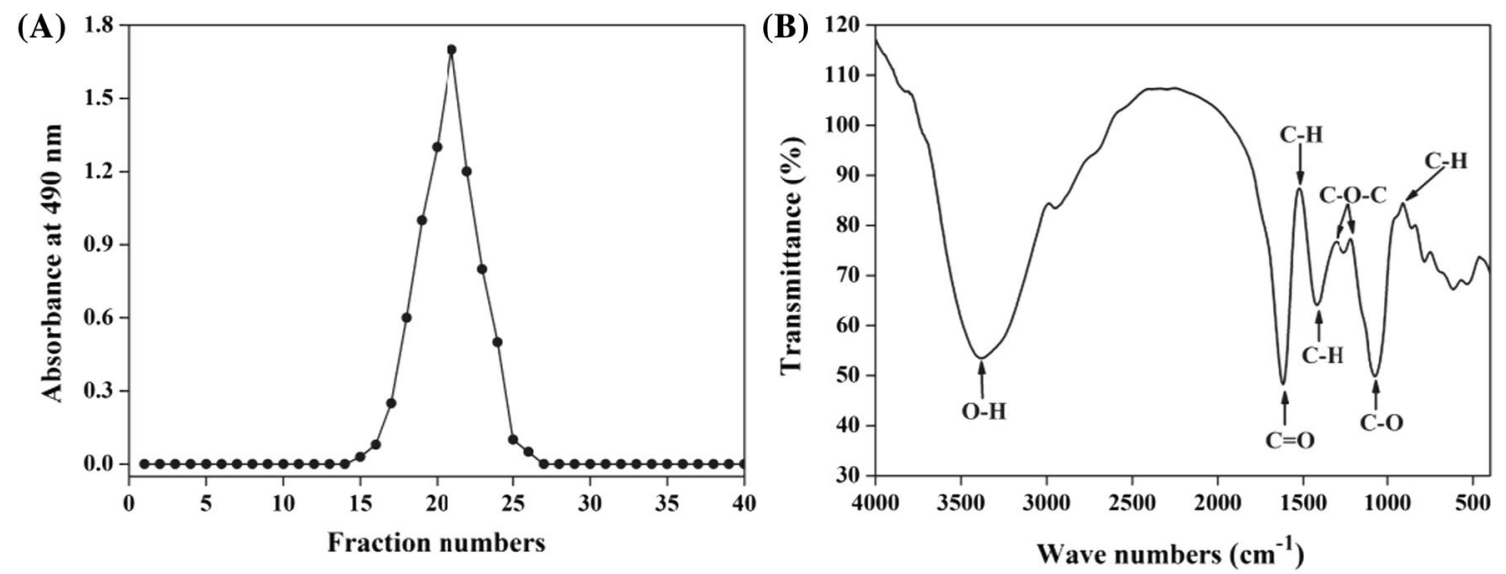

Fig. 5 Elution profiles (a) and FT-IR spectroscopy (b) of polysaccharide produced by C. globosum CGMCC 6882 from co-culture with G. pentaphyllum herb 
useful approaches for enhancing production through coculture with host plant or addition of elicitors.

Acknowledgements This work is supported by the Natural Science Foundation of Youth Support Plan of Henan University of Technology (2017QNJH10) and High Level Research Fund for Qualified People of Henan University of Technology (2017BS011), the Science and Technology Research Program of Chongqing Municipal Education Commission (KJQN201800540), the natural science foundation of Henan provincial education department (19A180015)

\section{Compliance with ethical standards}

Conflict of interest The authors declare that there are no conflicts of interest.

\section{References}

1. Lu B, Lu F, Ran L, Yu K, Xiao Y, Li Z, Dai F, Wu D, Lan G (2018) Self-assembly of natural protein and imidazole molecules on gold nanoparticles: applications in wound healing against multi-drug resistant bacteria. Int J Biol Macromol 119:505-516

2. Xu X, Nie S, Ding H, Hou FF (2018) Environmental pollution and kidney diseases. Nat Rev Nephrol 14:313

3. Alvin A, Miller KI, Neilan BA (2014) Exploring the potential of endophytes from medicinal plants as sources of antimycobacterial compounds. Microbiol Res 169(7):483-495

4. Mandal S, Mn M, Mandal SK (2009) Rational drug design. Eur J Pharmacol 625(1):90-100

5. Deepika VB, Murali TS, Satyamoorthy K (2016) Modulation of genetic clusters for synthesis of bioactive molecules in fungal endophytes: a review. Microbiol Res 182:125-140

6. Nisa H, Kamili AN, Nawchoo IA, Shafi S, Shameem N, Bandh SA (2015) Fungal endophytes as prolific source of phytochemicals and other bioactive natural products: a review. Microb Pathogenesis 82:50-59

7. Venugopalan A, Srivastava S (2015) Endophytes as in vitro production platforms of high value plant secondary metabolites. Biotechnol Adv 33(6, Part 1):873-887

8. Xie F, De Thaye E, Vermeulen A, Van Bocxlaer J, Colin P (2018) A dried blood spot assay for paclitaxel and its metabolites. J Pharmaceut Biom 148:307-315

9. Dan L, Tian L, Jian K, Ying Z, Hai-Feng W (2016) Synthesis and anti-tumor activities of 4-anilinoquinoline derivatives. Molecules 21(1):1-8

10. Seetharaman $P$, Gnanasekar S, Chandrasekaran R, Chandrakasan G, Kadarkarai M, Sivaperumal S (2017) Isolation and characterization of anticancer flavone chrysin (5,7-dihydroxy flavone)producing endophytic fungi from Passiflora incarnata L. leaves. Ann Microbiol 67(4):321-331

11. Kusari S, Pandey SP, Spiteller M (2013) Untapped mutualistic paradigms linking host plant and endophytic fungal production of similar bioactive secondary metabolites. Phytochemistry 91:81-87

12. Zhou JY, Sun K, Chen F, Yuan J, Li X, Dai CC (2018) Endophytic Pseudomonas induces metabolic flux changes that enhance medicinal sesquiterpenoid accumulation in Atractylodes lancea. Plant Physiol Biochem 130:473-481

13. Wang Z, Xue R, Cui J, Wang J, Fan W, Zhang H, Zhan X (2019) Antibacterial activity of a polysaccharide produced from Chaetomium globosum CGMCC 6882. Int J Biol Macromol 125:376-382
14. Wang Z, Wu J, Zhu L, Zhan X (2016) Activation of glycerol metabolism in Xanthomonas campestris by adaptive evolution to produce a high-transparency and low-viscosity xanthan gum from glycerol. Bioresour Technol 211:390-397

15. Yang LB, Zhan XB, Zheng ZY, Wu JR, Gao MJ, Lin CC (2014) A novel osmotic pressure control fed-batch fermentation strategy for improvement of erythritol production by Yarrowia lipolytica from glycerol. Bioresour Technol 151:120-127

16. Liu RS, Li DS, Li HM, Tang YJ (2008) Response surface modeling the significance of nitrogen source on the cell growth and Tuber polysaccharides production by submerged cultivation of Chinese truffle Tuber sinense. Process Biochem 43(8):868-876

17. Sarchami T, Johnson E, Rehmann L (2016) Optimization of fermentation condition favoring butanol production from glycerol by Clostridium pasteurianum DSM 525. Bioresour Technol 208:73-80

18. Zhu C, Fang B, Wang S (2016) Effects of culture conditions on the kinetic behavior of 1,3-propanediol fermentation by Clostridium butyricum with a kinetic model. Bioresour Technol 212:130-137

19. Kim IJ, Youn HJ, Kim KH (2016) Synergism of an auxiliary activity 9 (AA9) from Chaetomium globosum with xylanase on the hydrolysis of xylan and lignocellulose. Process Biochem 51(10):1445-1451

20. Umikalsom MS, Ariff AB, Karim MIA (1998) Saccharification of pretreated oil palm empty fruit bunch fiber using cellulase of Chaetomium globosum. J Agric Food Chem 46(8):3359-3364

21. Skriptsova AV (2017) Nitrogen effect on water-soluble polysaccharide accumulation in Streblonema sp. (Ectocarpales, Phaeophyceae). Mar Biotechnol 19(4):410-419

22. Jiang J, Sun YF, Tang X, He CN, Shao YL, Tang YJ, Zhou WW (2018) Alkaline $\mathrm{pH}$ shock enhanced production of validamycin A in fermentation of Streptomyces hygroscopicus. Bioresour Technol 249:234-240

23. Wu J, Zhang X, Zhou J, Dong M (2016) Efficient biosynthesis of (2S)-pinocembrin from D-glucose by integrating engineering central metabolic pathways with a $\mathrm{pH}$-shift control strategy. Bioresour Technol 218:999-1007

24. Zou J, Pan J, He H, Wu S, Xiao N, Ni Y, Li J (2018) Nitrifying aerobic granular sludge fermentation for releases of carbon source and phosphorus: the role of fermentation $\mathrm{pH}$. Bioresour Technol 260:30-37

25. Mai HTN, Lee KM, Choi SS (2016) Enhanced oxalic acid production from corncob by a methanol-resistant strain of Aspergillus niger using semi solid-sate fermentation. Process Biochem 51(1):9-15

26. Fang QH, Zhong JJ (2002) Submerged fermentation of higher fungus Ganoderma lucidum for production of valuable bioactive metabolites_-ganoderic acid and polysaccharide. Biochem Eng J 10(1):61-65

27. Bamisile BS, Dash CK, Akutse KS, Keppanan R, Afolabi OG, Hussain M, Qasim M, Wang L (2018) Prospects of endophytic fungal entomopathogens as biocontrol and plant growth promoting agents: an insight on how artificial inoculation methods affect endophytic colonization of host plants. Microbiol Res 217:34-50

28. Brader G, Compant S, Mitter B, Trognitz F, Sessitsch A (2014) Metabolic potential of endophytic bacteria. Curr Opin Biotech 27:30-37

29. Wang J, Yang JL, Zhou PP, Meng XH, Shi YP (2017) Further new gypenosides from Jiaogulan (Gynostemma pentaphyllum). J Agric Food Chem 65(29):5926-5934

30. Jiang C, Song J, Zhang J, Yang Q (2017) Identification and characterization of the major antifungal substance against Fusarium sporotrichioides from Chaetomium globosum. World J Microbiol Biotechnol 33(6):108

31. Hu Y, Zhang W, Zhang P, Ruan W, Zhu X (2013) Nematicidal activity of chaetoglobosin A poduced by Chaetomium globosum 
NK102 against Meloidogyne incognita. J Agric Food Chem 61(1):41-46

32. Zhong L, Niu B, Tang L, Chen F, Zhao G, Zhao J (2016) Effects of polysaccharide elicitors from endophytic Fusarium oxysporum Fat 9 on the growth, flavonoid accumulation and antioxidant property of Fagopyrum tataricum sprout cultures. Molecules 21(12): 1590

33. Tian Y, Amand S, Buisson D, Kunz C, Hachette F, Dupont J, Nay B, Prado S (2014) The fungal leaf endophyte Paraconiothyrium variabile specifically metabolizes the host-plant metabolome for its own benefit. Phytochemistry 108:95-101

34. Shweta S, Shivanna MB, Gurumurthy BR, Shaanker RU, Kumar TRS, Ravikanth G (2014) Inhibition of fungal endophytes by camptothecine produced by their host plant, Nothapodytes nimmoniana (Grahm) Mabb. (Icacinaceae). Curr Sci 107(6):994-1000

35. Yu Y, Shen M, Song Q, Xie J (2018) Biological activities and pharmaceutical applications of polysaccharide from natural resources: a review. Carbohydr Polym 183:91-101

Publisher's Note Springer Nature remains neutral with regard to jurisdictional claims in published maps and institutional affiliations. 